\title{
Implementation of a wireless energy management and safety system
}

\author{
Cheng-Chien Kuo ${ }^{\mathrm{a} *}$, Han-Ching Lin $^{\mathrm{b}}$ \\ ${ }^{a}$ Saint John's University, 499, Sec. 4, Tamking Rd., TamSui District, New Taipei City, Taiwan \\ ${ }^{b}$ Chihlee Institute of Technology, 313, Sec. 1, Wunhua Rd., Banciao District, New Taipei City, Taiwan
}

\begin{abstract}
The Energy Management and Safety System (EMSS) proposed in this paper uses ZigBee wireless sensor network in combination with the energy parameters measurement and the control of safety protection. The system consists of the intelligent outlet module, wireless transmission module and central monitor and control module. Combining with the energy monitor and safety protection software, the system can achieve seven major functions, which are energy usage monitor, energy usage control, standby power management, circuit loop energy monitor and protection control, outlet overheat protection, electric leakage protection and neutral line over-voltage protection. In addition, the all-graphic human machine interfaces is implemented to display the power usage and all control functions of electric equipment completely. The system can reduce energy losses effectively, and improve safety protection of electric equipment usage using the energy management and safety system proposed.
\end{abstract}

Keywords: Energy management and safety system, ZigBee, energy saving, intelligent outlet

\section{Introduction}

The excessive usage of fossil energy has led to the threat of energy crisis. Moreover, due to worldwide industrialization, the global climate becomes abnormal and mankind is facing severe problems of existence. Therefore, besides finding clean, environment-friendly and non-exhausted new energies, it is necessary to develop appropriate energy-saving technologies, in order to effectively slow down the energy usage and alleviate the severe changes of environment.

Many kinds of electric equipment are frequently used without being disconnected from the outlets [1][2]. The consumed standby power accumulated over a long period constitutes not only a kind of energy loss but also an extra expenditure for households. If the power, voltage, current, frequency and other energy parameters under both operation and standby status of an electric equipment could be obtained, it could be turned on and off by the energy management and safety system. The accumulation of saved energy by this way would be hard to ignore [3].

Extension line is a kind of very convenient electric equipment. However, the load of such kind of equipment is usually unknown [4] so in the case of unknown consuming powers of the electric equipment, repeated plugging in the electric equipment would be liable to cause the wires to catch fire. As shown in the recent fire statistics in Taiwan [5], 30\% of the fires were caused by electric equipment with the reasons mostly being extension line used in overload status or aging of electric equipment, components and wires along with impossible in-time response or no action in the case of overloading due to aging of no fuse breaker, which caused the wires to catch fire and resulted in those fires ultimately [6]-[8]. If the temperature sensors are installed through the system, they would automatically trip and disconnect the connection between the electric equipment and main power once the abnormal high temperature is detected in the case of overload or circuit anomalies thus accidents could be prevented. The electric

\footnotetext{
* Manuscript received March 14, 2015; revised July 23, 2015.

Corresponding author. Tel.: +886920881490; E-mail address: cckuo@mail.sju.edu.tw.

doi: 10.12720 /sgce.4.2.125-131
} 
leakage detection circuit and neutral line over-voltage detection circuit could detect the leakage of electric equipment at the first time and give out warning in advance or shut off the power supple to prevent users from touching the electric equipment without knowing the situation and occurrence of any dangers due to electric shocks.

Therefore, an integrated energy management and safety system combined with the functions of energy monitor and power control would be presented in the study. It could be used by households and office locations in any environments. Up to the high performance of easy expandability and convenience of overall system construction, it could save the unnecessary power consumption in households and improve the safety protection of household electric products so as to achieve the triple objectives on energy conservation and carbon reduction, energy monitor and safety protection.

\section{System Structure}

The proposed system is as shown in Fig. 1. As shown in the figure, each piece of electric equipment is plugged onto the designed intelligent outlet module. All measured energy parameters are transmitted through the Zigbee wireless transmission module to the central monitor and control module where analysis and discriminating are made for decisions uniformly through powerful computing then commands are sent out by the wireless communication module to the intelligent outlet module to take actions accordingly. However, since some power usage safety protection functions need rather fast reaction, for instance, in the cases of electric leakage, over temperature or neutral line over voltage, power supply of the outlet needs to be disconnected instantly. Therefore these functions must make immediate decisions and take fast actions directly within the intelligent outlet module and send back the results to the central monitor and control module for display or statistic need after the actions have been completed.

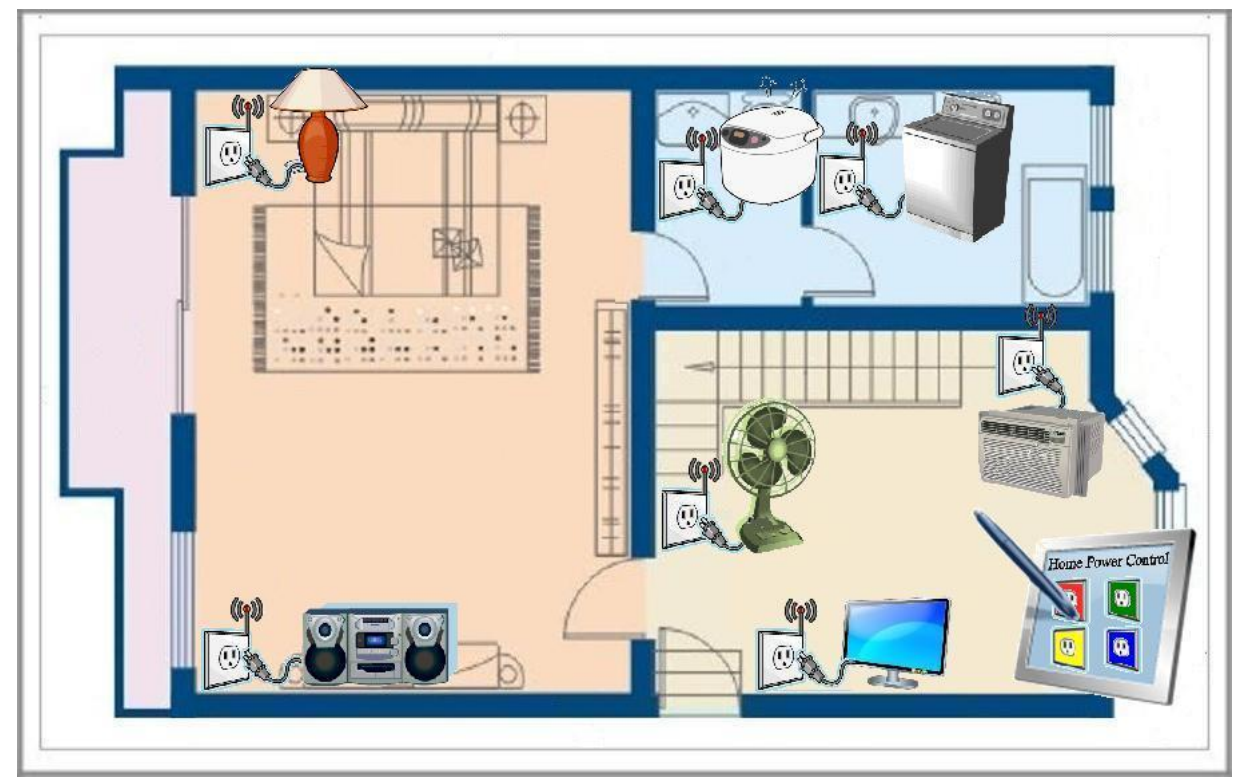

Fig. 1. Concept diagram of energy management and safety system.

The proposed system could be divided into three hardware modules, namely, the intelligent outlet module, wireless transmission module and central monitor and control module. The intelligent outlet module mainly accomplishes detection of energy status, outlet overheat protection and power supply control through the over temperature detection circuit and on-off circuit, power supply control, remote control through the wireless transmission module and transmission of the detected energy signal and outlet power supply status to the central monitor and control module via wireless transmission module. The central monitor and control module would analyze and calculate the transmitted energy parameter 
information and display it on the system interface. The energy usage control, standby power management and circuit loop energy monitor control could be realized through integration of each intelligent outlet module by the central monitor and control module and advanced setting by the software provider. Therefore seven software functions could be attained based on the hardware structure designed in this system. The complete system structure is as shown in Fig. 2.



Fig. 2. System structure of the proposed EMSS.

By adopting the master-slave structure, the system is divided into two parts, namely, the intelligent outlet module and central monitor and control module with the wireless transmission module connecting each part in between.

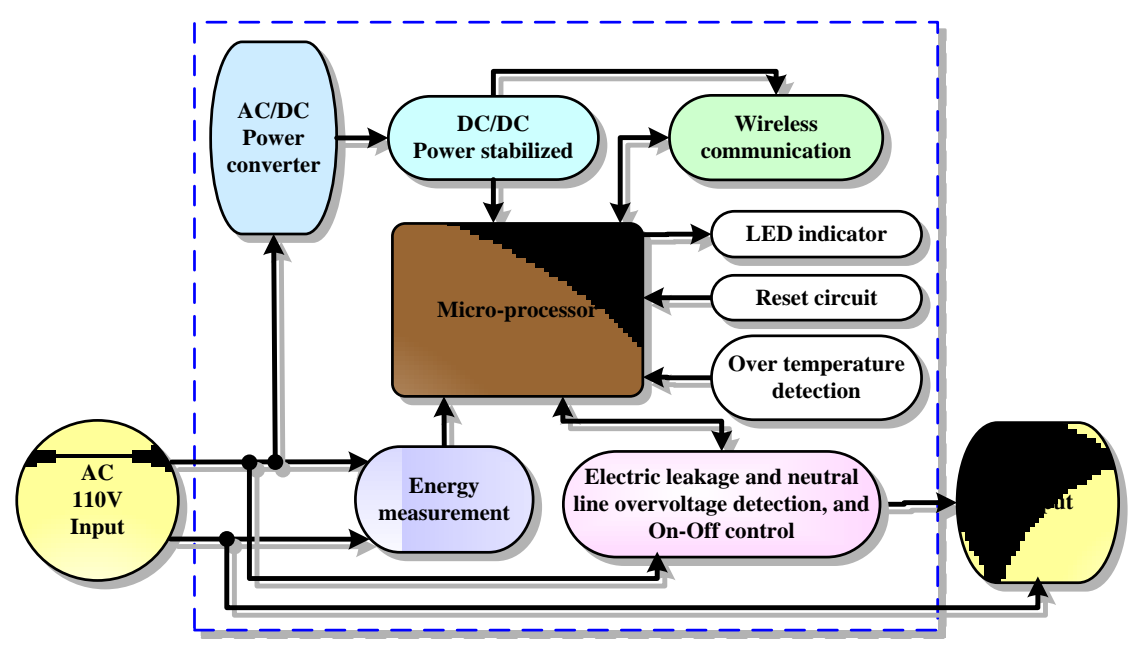

Fig. 3. Hardware diagram of intelligent outlet module.

\subsection{Intelligent outlet module}

The intelligent outlet module is a monitor and control end device with its hardware structure as shown in Fig. 3. The intelligent outlet module mainly consists of the power supply module, microprocessor unit, reset circuit unit, ZigBee wireless transmission module, neutral line over-voltage detection unit, energy measurement unit, over temperature detection unit, electric leakage detection unit and on-off control unit with the seven functions that could be realized including: 
1. Energy parameters measurement of loads at the outlet end

2. On-off control of power output at the outlet end

3. Temperature safety protection

4. Electric leakage detection and safety protection

5. Neutral line over-voltage safety protection

6. LED real-time display of power output status at the outlet end

7. Data transmission to central monitor and control module via ZigBee module

\subsection{Central monitor and control module}

The central monitor and control module is the data aggregation center of the intelligent outlet module as well as the transmission center of the discriminating and control commands. Information is transmitted and received through the wireless transmission module, which is connected to a USB interface through the touch panel computer unit. Its hardware structure is as shown in Fig. 4. The central monitor and control module mainly including the touch panel computer unit, USB transmission unit, AC/DC power stabilized unit and ZigBee wireless transmission module can majorly realize the follow three function:

1. Energy parameters display and control interface

2. Information transmission of touch panel computer unit and ZigBee wireless transmission module

3. Transmission and receiving information by the intelligent outlet module via ZigBee



Fig. 4. Hardware diagram of central monitor and control module.

\section{System Implementation}

The designed outlet module could be put into the simple commercial outlet box made of transparent plastic and standard specifications, which could be plugged in directly for the use in old building or recessed in the wall-mounted outlet box for the use in new buildings. Fig. 5 shows the proposed intelligent outlet module placed in a commercial outlet box.



Fig. 5. The proposed intelligent outlet module placed in commercial outlet box. 
The whole energy management and safety system is made up by the aforementioned systems, equipment and the touch panel computer. In this study, several intelligent outlet modules and coordinators have been constructed to carry out the hardware test and system experiments with the whole proposed system as shown in Fig. 6.

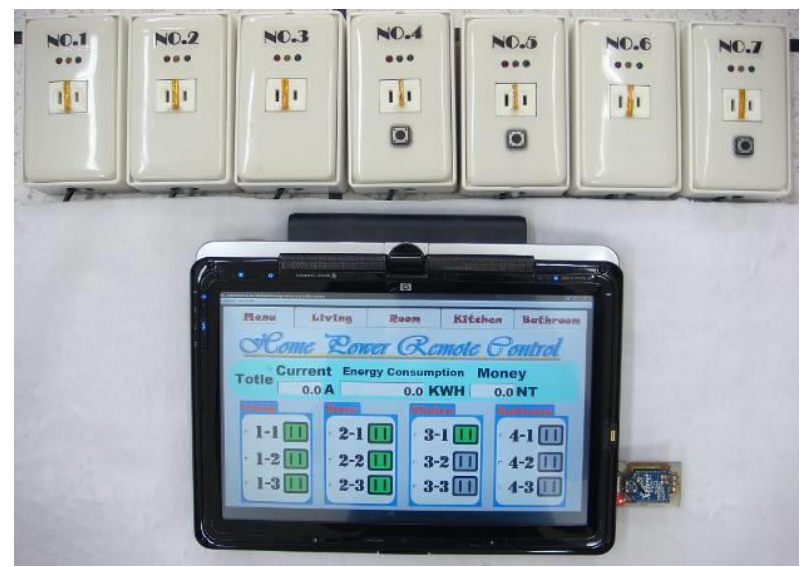

Fig. 6. The whole proposed system.

The proposed energy management and safety system designed from the perspective of users could be applied in various interior environments due to its flexible configuration methods. With the simplified graphical operation interfaces, users could grasp the three major functions of energy saving, monitor and control and safety without costing too much time in installation and study. The monitor and control could be carried out through the central monitor and control module immediately after the setup of the hardware. Within the control software, the main page, each spatial page, intelligent setup, loop protection and other major and functional pages are in place and can be operated by the touch-panel method to conduct setup and operation of the system. All intelligent outlet module status are provided in the main page so that the on-off status, timer setting on-off control of each outlet and power usage status of the whole outlet systems under monitor and control could be known by the display of graphics and colors and approximate fees of overall power usage could be estimated. Fig. 7 shows the main page of EMSS.

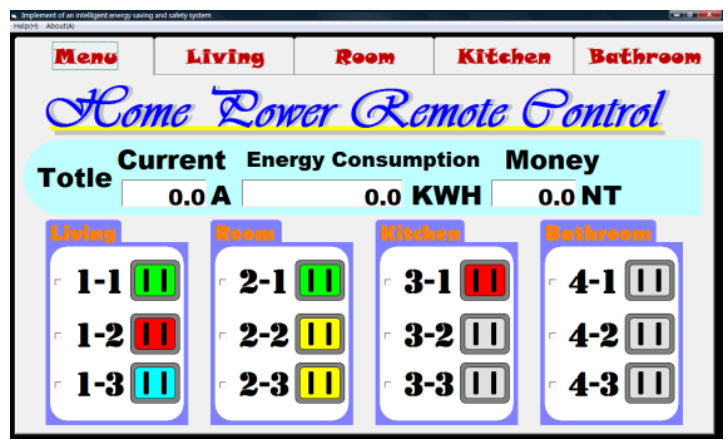

Fig. 7. Main page of EMSS.

In Fig. 7, outlets are displayed in the main page representing the intelligent outlet modules in 4 spatial pages, in which 3 outlet systems are controlled respectively. Each graphic is displayed according to the setup status on each subordinate page and the back transmission state of the outlet system. The graphic is indicated in grey color when the outlet system is not energized and used and indicated in green color when the outlet system is involved in normal actions to inform the users that the outlet system is in normal power supply status. When the graphic is indicated in red color, it means that current outlet system is disconnected and not supplying power either because of the power supply disconnection by the 
user through the remote on-off control function or protection control by trip or automatic power disconnection when the outlet system detects over temperature or overload. In this case, if it is not set up or controlled by the user then the outlet system indicated in red color must be checked to verify and eliminate the failures or abnormities before the power supply is resumed. When the graphic of outlet is indicted in yellow or blue color, it means the outlet system is under timer setting on-off control by the user where the yellow indicates the timer setting off control and the blue color indicates the timer setting on control so that the users could easily discriminate the current status of each outlet system by different colors.

There are 4 preset spatial pages, namely living room, room, kitchen and bathroom. After equipment numbering of each intelligent outlet module is completed, the sequencing will be carried out based on the equipment numbering starting from the first page, for example, the living room is number as No. 1 to No.3 outlet systems, the room is numbered as No. 4 to No. 6 and the rest can be done in the small manner. Total current, total power and rough estimation of power usage fees of each space are provided in each spatial page and four kinds of energy parameters of voltage, current, power and frequency are provided for each outlet system for the users to monitor the outlet usage status all the time and take remote on-off control. The users only need to put their fingers on the central monitor and control module to turn on or off the electric equipment. Same outlet status graphics of the outlet system will also be provided on the main page so the users could identify the setup status of the outlet systems by colors. Advanced intelligent settings and loop protection selection control will be set up from this page. The spatial page is as shown in Fig. 8.

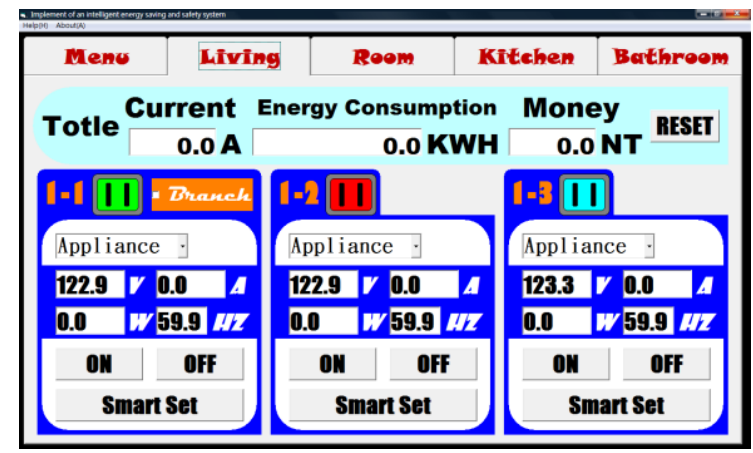

Fig. 8. Spatial page of EMSS.

\section{Conclusion}

In this paper, a set of energy management and safety system has been successfully accomplished, which primarily achieves the detection of the outlet power supply status during the usage of electric equipment loads and ordinary times with its automation and safety being further improved by the microprocessor and other surrounding circuits. Combined with ZigBee wireless transmission function, it offers convenience for remote monitoring of the status of each monitoring point, effectively elevates the quality and convenience of power usage and smoothly improves the situation where power usage was unknown to traditional outlets thus users are able to grasp the power usage status through displayed values, take correct protection to the lines to avoid overload and prevent from property losses and personal injuries due to anomalies or overload of the electric equipment and has certain socioeconomic benefits and vast development prospects. Energy monitor is the most effective method of energy saving and safety protection. Generally, when the electric equipment are not in usage, most of the power consumption is standby power consumption that could be eliminated. Without the aid of observation through the monitor and control system, the accumulated power consumption during electric equipment standby period is hard to be perceived. Also, the continuous loading on the same loop under unknown circumstance would result in not only unnecessary energy loss but also major hazards. By using the 
energy monitor system and automatic protection lines, the overload could be prevented. Disconnecting the standby electric equipment automatically could ensure the power usage safety and bring in benefits in energy saving. The feature of ZigBee in supporting to a large number of nodes is benefit for its future expansion where the newly migrated intelligent outlet nodes could combine with the original system immediately. Its convenience in setup and expansion meets the requirements of various power usage environments. A graphic monitor and control system is constructed by the central monitor and control module by using Visual Basic software. With the combination of the touch panel computer setup and easy operation interfaces, it is easy for operation by anybody and realizes the all-time monitor and control in terms of energy saving and safety.

The application of the energy management and safety system has improved the quality of electric usage of the households and offices through which the power could be reviewed through values and controlled at any time and elevated the level of power usage safety. Therefore, it not only contributes to the energy conservation and carbon reduction but also improves living quality and safety.

\section{Acknowledgements}

The support of this research by the Ministry of Science and Technology of the Republic of China under Grant No. MOST103-2632-E-129-002-MY3 and MOST103-2221-E-129-003 are gratefully acknowledged.

\section{References}

[1] Song G, Ding F, Zhang W, Song A. A wireless power outlet system for smart homes. IEEE Transactions on Consumer Electronics, 2008;54(4):1688-1691.

[2] Song G, Zhou Y, Zhang W, Song A. A multi-interface gateway architecture for home automation networks. IEEE Transactions on Consumer Electronics, 2008;54(3):1110-1113.

[3] Lien CH, Lin CH, Bai YW, Liu MF, Lin MB. Remotely controllable outlet system for home power management. In: Proc. of IEEE Tenth International Symposium on Consumer Electronics, 2006:1-6.

[4] Lien CH, Bai YW, Lin MB. Remote-controllable power outlet system for home power management. IEEE Transactions on Consumer Electronics, 2007;53(4):1634-1641.

[5] Lien CH, Chen HC, Bai YW, Lin MB. Power monitoring and control for electric home appliances based on power line communication. In: Proc. of IEEE Instrumentation and Measurement Technology Conference, 2008:2179-2184.

[6] Han J, Lee H, Park KR. Remote-controllable and energy-saving room architecture based on ZigBee communication. IEEE Transactions on Consumer Electronics, 2009;5(1):264-268.

[7] Lee WS, Hong SH. KNX - ZigBee gatway for home automation. In: Proc. of IEEE International Conference on Automation Science and Engineering, 2008:750-755.

[8] Gill K, Yang SH, Yao F, Lu X. A ZigBee-based home automation system. IEEE Transactions on Consumer Electronics, 2009; 55(2):422-430. 